\title{
Coupled Task-Space Admittance Controller Using Dual Quaternion Logarithmic Mapping
}

DOI:

10.1109/LRA.2020.3010458

\section{Document Version}

Accepted author manuscript

Link to publication record in Manchester Research Explorer

\section{Citation for published version (APA):}

Fonseca, M. D. P. A., Adorno, B. V., \& Fraisse, P. (2020). Coupled Task-Space Admittance Controller Using Dual Quaternion Logarithmic Mapping. IEEE Robotics and Automation Letters, 5(4), 6057-6064.

https://doi.org/10.1109/LRA.2020.3010458

\section{Published in:}

IEEE Robotics and Automation Letters

\section{Citing this paper}

Please note that where the full-text provided on Manchester Research Explorer is the Author Accepted Manuscript or Proof version this may differ from the final Published version. If citing, it is advised that you check and use the publisher's definitive version.

\section{General rights}

Copyright and moral rights for the publications made accessible in the Research Explorer are retained by the authors and/or other copyright owners and it is a condition of accessing publications that users recognise and abide by the legal requirements associated with these rights.

\section{Takedown policy}

If you believe that this document breaches copyright please refer to the University of Manchester's Takedown Procedures [http://man.ac.uk/04Y6Bo] or contact uml.scholarlycommunications@manchester.ac.uk providing relevant details, so we can investigate your claim.

\section{OPEN ACCESS}




\title{
Coupled Task-Space Admittance Controller Using Dual Quaternion Logarithmic Mapping
}

\author{
Mariana de Paula Assis Fonseca, ${ }^{1}$ Bruno Vilhena Adorno, ${ }^{1}$ and Philippe Fraisse ${ }^{2}$
}

\begin{abstract}
This paper proposes a six-DOF task-space admittance controller using the dual quaternion logarithmic mapping, coupling the translation and rotation impedance in a single mathematical structure. The controller is designed based on the energy of the system and the stiffness matrix is build to be consistent with the task geometry. Moreover, the formulation is free of topological obstruction and we present a solution for the unwinding phenomenon based on a switched error function. The closed-loop system is composed of an inner motion control loop to ensure the trajectory tracking of the end-effector pose while an outer loop imposes a desired apparent impedance to the robot. Experiments executed on a KUKA LWR4+ robot with a force/torque sensor in the end-effector, together with statistical analyses, show better performance of the proposed controller over one of the main six-DOF controllers from the state of the art. More specifically, our controller presents an exponential decay in all situations, a task-error closed-loop behavior closer to the desired one, and it is free from topological obstruction and unwinding, while presenting a statistically equivalent control effort.
\end{abstract}

Index Terms-Compliance and Impedance Control; Physical Human-Robot Interaction

\section{INTRODUCTION}

W HEN a robot interacts with the environment, contact wrenches may appear. For a safe interaction, it is crucial to ensure a compliant robot behavior, which can be imposed by controlling its apparent impedance [1], [2]. Considering the execution of six-degree-of-freedom (DOF) tasks, the end-effector pose (position and orientation) must be handled, and the mechanical impedance ought to be defined to have a stiffness that is physically consistent with the task geometry to prevent unnatural behavior [2]. Hence, a suitable representation of the end-effector rotation related to the contact moment must be used [1].

In classic approaches, position and orientation are uncoupled in the control law and the orientation is usually based on minimal representations, such as the Euler angles, which have

Manuscript received: February, 24, 2020; Revised May, 18, 2020; Accepted July, 04, 2020.

This paper was recommended for publication by Editor Paolo Rocco upon evaluation of the Associate Editor and Reviewers' comments. This work was supported by the Brazilian agency CAPES, CNPq (grant numbers 424011/2016-6 and 303901/2018-7), and by the French agency CNRS.

${ }^{1}$ M. P. A. Fonseca is with the Graduate Program in Electrical Engineering and B. V. Adorno is with the Department of Electrical Engineering of the Universidade Federal de Minas Gerais (UFMG) - Av. Antônio Carlos 6627, 31270-901, Belo Horizonte-MG, Brazil. Emails: maripaf@gmail . com and adornodieee. org

${ }^{2} \mathrm{P}$. Fraisse is with LIRMM UMR 5506 CNRS - Université de Montpellier (UM) - 161 rue Ada Montpellier, 34392, Montpellier, France. Email: philippe.fraissedlirmm. fr

Digital Object Identifier (DOI): see top of this page. representation singularities [3] and do not lead to a physically meaningful impedance [1]. Caccavale et al. propose to use an energy-based argument to develop an impedance equation, and to use the imaginary part of a unit quaternion for the orientation displacement, expressing the mutual orientation between the compliant and the desired frames. However, they use different control laws for the position and orientation and their controller presents a stiffness that is geometrically consistent only for infinitesimal displacements [1]. Furthermore, their formulation presents the topological obstruction problem as an unstable equilibrium point.

Caccavale et al. [2] extend that work [1] to propose a controller where the stiffness is geometrically consistent not only for infinitesimal displacements but also for finite ones. However, the new controller still presents the topological obstruction problem.

We recently have proposed a coupled six-DOF admittance controller using the dual quaternion (DQ) logarithm [4]. ${ }^{1}$ Although that controller is simple and the experimental results have shown its effectiveness, we have not shown that the closed-loop system is stable. Also, poor choices of parameters, most notably of the stiffness matrix, may result in a controller that do not have a physical meaning and is not geometrically consistent with the task. Another drawback of that controller is the unwinding phenomenon, where the end-effector pose may be close to the desired pose and yet rotate through large angles before reaching the equilibrium [5]. This current paper addresses all the aforementioned problems.

\section{A. Statement of contributions}

The main contribution of this work is a new six-DOF admittance controller based on the DQ logarithmic mapping of the task-space displacement that has the following features:

1) the stiffness term is designed to be geometrically consistent with the six-DOF task, which makes the controller have a physical meaning;

2) the DQ logarithmic mapping has shown an exponential decay of the error norm in all situations of free-motion thanks to the linearity of the stiffness term, which is not the case if the imaginary part of the unit quaternion is used to represent the rotation, as in [2];

3 ) it does not have the problem of topological obstruction, as in [2], and a solution for the unwinding phenomenon is proposed based on a switching error function that

\footnotetext{
${ }^{1}$ That work was presented as an extended abstract in the Workshop Applications of Dual Quaternion Algebra to Robotics, which happened at ICAR 2019
} 
maps the two positive invariant sets (PIS) of the space of unit DQ into a single PIS in the image of the logarithmic mapping. Moreover, the trajectories of the closed-loop system always converge to that single PIS without exhibiting chattering, which would require a more complex hybrid control strategy [6];

4) the closed-loop system is proven to be passive and thus stable.

Experiments are done in a KUKA LWR4+ robot and statistical analyses are performed to compare our approach with the state of the art.

\section{MATHEMATICAL BACKGROUND}

Dual quaternions have a compact representation, do not have representational singularities, and their coefficients can be directly used in the proposed control law. Also, they have strong algebraic properties and can be used to represent rigid motions, twists, wrenches, and several geometrical primitives such as planes, lines, etc. Furthermore, the extraction of geometric parameters such as translation, rotation axis, and rotation angle is very simple [7].

Quaternions can be understood as an extension of imaginary numbers, where the three imaginary components obey $\hat{\imath}^{2}=\hat{\jmath}^{2}=\hat{k}^{2}=\hat{\imath} \hat{\jmath} \hat{k}=-1$. The set of quaternions is defined as $\mathbb{H} \triangleq\left\{h_{1}+\hat{\imath} h_{2}+\hat{\jmath} h_{3}+\hat{k} h_{4}: h_{1}, h_{2}, h_{3}, h_{4} \in \mathbb{R}\right\}$, such that, given $\boldsymbol{h} \in \mathbb{H}$, the real part is $\operatorname{Re}(\boldsymbol{h}) \triangleq h_{1}$ and $\operatorname{Im}(\boldsymbol{h}) \triangleq \hat{\imath} h_{2}+\hat{\jmath} h_{3}+\hat{k} h_{4}$ is the imaginary part. The subset of pure quaternions $\mathbb{H}_{p} \subset \mathbb{H}$ contains the elements whose real part equals zero and the subset of unit quaternions $\mathbb{S}^{3} \subset \mathbb{H}$ contains only elements with unit norm. Similarly, the DQ set is defined as $\mathcal{H} \triangleq\left\{\boldsymbol{h}_{1}+\varepsilon \boldsymbol{h}_{2}: \boldsymbol{h}_{1}, \boldsymbol{h}_{2} \in \mathbb{H}, \varepsilon \neq 0, \varepsilon^{2}=0\right\}$, where $\varepsilon$ is the nilpotent dual unit. The subset of pure DQ, $\mathcal{H}_{p} \subset \mathcal{H}$, contains only elements whose real part equals zero and the subset of unit DQ, $\underline{\mathcal{S}} \subset \mathcal{H}$, contains only elements with unit norm.

\section{A. Dual quaternion logarithm and its relations}

Considering a translation $\boldsymbol{p}=\left(\hat{\imath} p_{x}+\hat{\jmath} p_{y}+\hat{k} p_{z}\right) \in \mathbb{H}_{p}$ and a rotation $\boldsymbol{r}=(\cos (\phi / 2)+\boldsymbol{n} \sin (\phi / 2)) \in \mathbb{S}^{3}$, with $\phi$ being the rotation angle around the rotation axis $\boldsymbol{n}=\left(\hat{\imath} n_{x}+\hat{\jmath} n_{y}+\hat{k} n_{z}\right) \in \mathbb{S}^{3} \cap \mathbb{H}_{p}$, the unit DQ that combines both $\boldsymbol{p}$ and $\boldsymbol{r}$ can be given by $\underline{\boldsymbol{x}}=(\boldsymbol{r}+(1 / 2) \varepsilon \boldsymbol{p} \boldsymbol{r}) \in \underline{\mathcal{S}}$, whose logarithm is $\mathcal{H}_{p} \ni \log \underline{\boldsymbol{x}}=(\boldsymbol{n} \phi+\varepsilon \boldsymbol{p}) / 2$ [8]. Moreover, the inverse of $\underline{\boldsymbol{x}}$ is given by $\underline{\boldsymbol{x}}^{*}$ such as $\underline{\boldsymbol{x}}^{*} \underline{\boldsymbol{x}}=\underline{\boldsymbol{x} \boldsymbol{x}^{*}}=1$.

The DQ logarithmic mapping can be used to translate the spacial difference $\underline{\tilde{x}}$ between two unit dual quaternions to the origin [9]. Considering $\underline{\tilde{\boldsymbol{x}}} \triangleq \underline{\boldsymbol{x}}^{*} \underline{\boldsymbol{x}}_{d}$, where $\underline{\boldsymbol{x}}, \underline{\boldsymbol{x}}_{d} \in \underline{\mathcal{S}}$ represent poses, $\underline{\boldsymbol{x}} \rightarrow \underline{\boldsymbol{x}}_{d}$ implies $\underline{\tilde{\boldsymbol{x}}} \rightarrow 1$, which implies $\log \underline{\tilde{\boldsymbol{x}}} \rightarrow 0$.

Letting $\underline{\boldsymbol{y}} \triangleq \log \underline{\boldsymbol{x}}$, the time derivatives of $\underline{\boldsymbol{y}}$ and $\underline{\boldsymbol{x}}$ are related by means of the matrix $\boldsymbol{Q}_{8}(\underline{\boldsymbol{x}})$ as [10]

$$
\operatorname{vec}_{8} \underline{\dot{\boldsymbol{x}}}=\boldsymbol{Q}_{8}(\underline{\boldsymbol{x}}) \operatorname{vec}_{6} \underline{\dot{\boldsymbol{y}}},
$$

where $\operatorname{vec}_{6}: \mathcal{H}_{p} \rightarrow \mathbb{R}^{6}$ and vec $_{8}: \mathcal{H} \rightarrow \mathbb{R}^{8}$.

Furthermore, there exists $\boldsymbol{E}(\underline{\boldsymbol{x}}) \in \mathbb{R}^{6 \times 6}$ such that

$$
\operatorname{vec}_{6} \underline{\boldsymbol{\zeta}}=\boldsymbol{E}(\underline{\boldsymbol{x}}) \operatorname{vec}_{6} \underline{\boldsymbol{y}},
$$

where $\boldsymbol{\zeta}=(\boldsymbol{\omega}+\varepsilon \boldsymbol{v}) \in \mathcal{H}_{p}$, with $\boldsymbol{\omega}, \boldsymbol{v} \in \mathbb{H}_{p}$ being the angular and the linear velocities, respectively. More specifically, since there exists a matrix $\boldsymbol{Q}_{4}(\boldsymbol{r}) \in \mathbb{R}^{4 \times 3}$ such that $\operatorname{vec}_{4} \dot{\boldsymbol{r}}=\boldsymbol{Q}_{4}(\boldsymbol{r}) \frac{d}{d t} \operatorname{vec}_{3}(\boldsymbol{n} \phi / 2)[10]$, where $\operatorname{vec}_{3}: \mathbb{H}_{p} \rightarrow \mathbb{R}^{3}$ and $\mathrm{vec}_{4}: \mathbb{H} \rightarrow \mathbb{R}^{4}$, and $\boldsymbol{\omega}=2 \dot{\boldsymbol{r}} \boldsymbol{r}^{*}$ [8], we find by inspection

$$
\boldsymbol{E}(\underline{\boldsymbol{x}}) \triangleq\left[\begin{array}{cc}
\overline{\boldsymbol{I}} \overline{\boldsymbol{W}}(\boldsymbol{r}) & \mathbf{0}_{3 \times 3} \\
\mathbf{0}_{3 \times 3} & 2 \boldsymbol{I}_{3 \times 3}
\end{array}\right],
$$

with $\mathbb{R}^{4 \times 3} \ni \overline{\boldsymbol{W}}(\boldsymbol{r}) \triangleq 2 \overline{\boldsymbol{H}}_{4}\left(\boldsymbol{r}^{*}\right) \boldsymbol{Q}_{4}(\boldsymbol{r})$ and $\overline{\boldsymbol{I}} \triangleq$ $\left[\begin{array}{ll}\mathbf{0}_{3 \times 1} & \boldsymbol{I}_{3 \times 3}\end{array}\right]$, where $\boldsymbol{I}_{n \times n} \in \mathbb{R}^{n \times n}$ is the identity matrix, $\mathbf{0}_{n \times m} \in \mathbb{R}^{n \times m}$ is a matrix of zeros, and $\overline{\boldsymbol{H}}_{4}(\cdot) \in \mathbb{R}^{4 \times 4}$ is an operator that satisfies $\operatorname{vec}_{4}(\boldsymbol{a} \boldsymbol{b})=\overline{\boldsymbol{H}}_{4}(\boldsymbol{b}) \operatorname{vec}_{4} \boldsymbol{a}$ [8].

Theorem 1. The inverse of (3) is given by

$$
\boldsymbol{E}^{-1}(\underline{\boldsymbol{x}}) \triangleq\left[\begin{array}{cc}
\frac{1}{2} \boldsymbol{Q}_{4}^{+}(\boldsymbol{r}) \overline{\boldsymbol{H}}_{4}(\boldsymbol{r}) \overline{\boldsymbol{I}}^{T} & \mathbf{0}_{3 \times 3} \\
\mathbf{0}_{3 \times 3} & \frac{1}{2} \boldsymbol{I}_{3 \times 3}
\end{array}\right],
$$

where $\boldsymbol{Q}_{4}^{+}(\cdot)$ is the left pseudo-inverse of $\boldsymbol{Q}_{4}(\cdot)$.

Proof: By direct calculation of $2 \overline{\boldsymbol{H}}_{4}\left(\boldsymbol{r}^{*}\right) \boldsymbol{Q}_{4}(\boldsymbol{r})$, we find, for all $r \in \mathbb{S}^{3}$,

$$
\overline{\boldsymbol{W}}(\boldsymbol{r})=\left[\begin{array}{c}
\mathbf{0}_{1 \times 3} \\
\boldsymbol{W}(\boldsymbol{r})
\end{array}\right]
$$

where $\boldsymbol{W}(\boldsymbol{r}) \in \mathbb{R}^{3 \times 3}$. Also, since $\operatorname{rank} \overline{\boldsymbol{H}}_{4}\left(\boldsymbol{r}^{*}\right)=4$ and $\operatorname{rank} \boldsymbol{Q}_{4}(\boldsymbol{r})=3$ for all $\boldsymbol{r} \in \mathbb{S}^{3}$ [10], from Corollary 2.5.10 of [11] we have that

$$
\operatorname{rank} \boldsymbol{A}+\operatorname{rank} \boldsymbol{B}-4 \leq \operatorname{rank} \boldsymbol{A} \boldsymbol{B} \leq \min \{\operatorname{rank} \boldsymbol{A}, \operatorname{rank} \boldsymbol{B}\}
$$

with $\boldsymbol{A} \triangleq \overline{\boldsymbol{H}}_{4}\left(\boldsymbol{r}^{*}\right)$ and $\boldsymbol{B} \triangleq \boldsymbol{Q}_{4}(\boldsymbol{r})$. Hence, rank $\overline{\boldsymbol{W}}(\boldsymbol{r})=3$ for all $\boldsymbol{r} \in \mathbb{S}^{3}$ and thus $\operatorname{rank} \boldsymbol{W}(\boldsymbol{r})=3$. Therefore, $\overline{\boldsymbol{I}} \overline{\boldsymbol{W}}(\boldsymbol{r})=\boldsymbol{W}(\boldsymbol{r})$ is full rank, which implies that for all $\boldsymbol{r} \in \mathbb{S}^{3}$ the inverse of $\boldsymbol{W}(\boldsymbol{r})$ exists and is given by $\boldsymbol{W}^{-1}=(1 / 2) \boldsymbol{Q}_{4}^{+}(\boldsymbol{r}) \overline{\boldsymbol{H}}_{4}(\boldsymbol{r}) \overline{\boldsymbol{I}}^{T}$. Indeed, since $\overline{\boldsymbol{I}}^{T} \overline{\boldsymbol{I}} \overline{\boldsymbol{W}}=\overline{\boldsymbol{W}}$ then

$$
\boldsymbol{W}^{-1} \boldsymbol{W}=\frac{1}{2} \boldsymbol{Q}_{4}^{+}(\boldsymbol{r}) \overline{\boldsymbol{H}}_{4}(\boldsymbol{r}) \overline{\boldsymbol{I}}^{T} \overline{\boldsymbol{I}} \overline{\boldsymbol{W}}(\boldsymbol{r})=\boldsymbol{I}
$$

because $\overline{\boldsymbol{H}}_{4}(\boldsymbol{r})=\overline{\boldsymbol{H}}_{4}\left(\boldsymbol{r}^{*}\right)^{-1}$ and $\boldsymbol{Q}_{4}^{+}(\boldsymbol{r}) \boldsymbol{Q}_{4}(\boldsymbol{r})=\boldsymbol{I}$ [10]. Moreover, as $\boldsymbol{W}$ is square and full rank, the left inverse equals the right inverse-i.e. $\boldsymbol{W}^{-1} \boldsymbol{W}=\boldsymbol{W} \boldsymbol{W}^{-1}=\boldsymbol{I}$. Consequently, $\boldsymbol{E}(\underline{\boldsymbol{x}})$ is also full rank and thus invertible.

\section{Control Strategies}

Given the desired pose $\underline{\boldsymbol{x}}_{d} \in \underline{\mathcal{S}}$ of an end-effector that interacts with the environment, we consider another (reference) frame specified by $\underline{\boldsymbol{x}}_{r} \in \underline{\mathcal{S}}$ such that a desired apparent impedance can be imposed on the pose displacements between $\underline{\boldsymbol{x}}_{d}$ and $\underline{\boldsymbol{x}}_{r}$ [1]. The closed-loop system is composed of a motion controller in the inner loop to control the endeffector pose according to the reference trajectory $\underline{\boldsymbol{x}}_{r}(t)$, while imposing a desired impedance behavior in the outer loop, as illustrated in Fig. 1. 


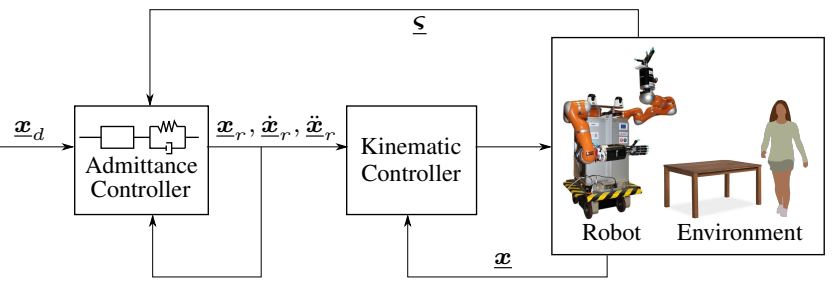

Figure 1: Scheme illustrating the control law composed of an outer loop with an impedance behavior and an inner loop with a motion controller. The wrench $\varsigma$ measured by the force/torque sensor at the robot end-effector is a result of the interaction with the environment.

\section{A. Admittance using the logarithmic mapping (ACLog)}

To impose the desired apparent impedance behavior to the robot, we first define an impedance control law that is physically meaningful and a stiffness matrix that is consistent with the task geometry. Similarly to what have been done in [1], [2], we derive the impedance equation based on the energy of the system, but using elements of dual quaternion algebra.

Consider a desired positive definite inertia matrix $\boldsymbol{M}_{d}=\operatorname{diag}\left(\boldsymbol{\mathcal { I }}_{3 \times 3}, m \boldsymbol{I}_{3 \times 3}\right)$, where $m$ is the mass and $\mathcal{I}_{3 \times 3} \in \mathbb{R}^{3 \times 3}$ is the inertia tensor. The kinetic energy of the system is given by

$$
\mathcal{K}=\frac{1}{2}\left(\operatorname{vec}_{6} \underline{\boldsymbol{\zeta}}_{r d}^{r}\right)^{T} \boldsymbol{M}_{d} \operatorname{vec}_{6} \underline{\boldsymbol{\zeta}}_{r d}^{r},
$$

where $\underline{\boldsymbol{\zeta}}_{r d}^{r}=\boldsymbol{\omega}_{r d}^{r}+\varepsilon \boldsymbol{v}_{r d}^{r}$ is the DQ with the angular and linear velocities from frame $\mathcal{F}_{r}$ to $\mathcal{F}_{d}$, with respect to frame $\mathcal{F}_{r}$.

To obtain the power, we take the time derivative of (5), which is given by

$$
\dot{\mathcal{K}}=\left(\operatorname{vec}_{6} \underline{\boldsymbol{\zeta}}_{r d}^{r}\right)^{T} \boldsymbol{I}^{\#} \operatorname{vec}_{6} \underline{\boldsymbol{\varsigma}}_{I}^{r},
$$

where $\operatorname{vec}_{6} \underline{\boldsymbol{S}}_{I}^{r} \triangleq \boldsymbol{I}^{\#} \boldsymbol{M}_{d} \operatorname{vec}_{6} \underline{\boldsymbol{\zeta}}_{r d}^{r}$ is the inertial wrench with respect to the frame $\mathcal{F}_{r}$ and

$$
\boldsymbol{I}^{\#} \triangleq\left[\begin{array}{ll}
\mathbf{0}_{3 \times 3} & \boldsymbol{I}_{3 \times 3} \\
\boldsymbol{I}_{3 \times 3} & \mathbf{0}_{3 \times 3}
\end{array}\right] .
$$

We also consider a dissipative damping wrench given by $\operatorname{vec}_{6} \underline{\boldsymbol{\varsigma}}_{D}^{r} \triangleq \boldsymbol{I}^{\#} \boldsymbol{B}_{d} \operatorname{vec}_{6} \underline{\boldsymbol{\zeta}}_{r d}^{r}$, with $\mathbb{R}^{6 \times 6} \ni \boldsymbol{B}_{d}>0$.

Now let us give special attention to the stiffness matrix regarding geometric consistency. Considering a positive definite stiffness matrix $\boldsymbol{K} \in \mathbb{R}^{6 \times 6}$, it can be decomposed as $\boldsymbol{K}=\boldsymbol{U} \boldsymbol{\Gamma} \boldsymbol{U}^{T}$ [12], with $\boldsymbol{\Gamma}=\operatorname{diag}\left(\gamma_{1}, \cdots, \gamma_{6}\right)$ in which $\gamma_{i}$, $i \in\{1, \ldots, 6\}$ represent the stiffnesses along the principal axes $\boldsymbol{u}_{i}$, which are the column vectors of the orthogonal matrix $\boldsymbol{U} \in \mathrm{O}(6)$. Therefore, the stiffness matrix can be specified with respect to a frame with the origin at the center of stiffness (i.e., the equilibrium point when there is no deformation), in terms of the stiffness parameters $\gamma_{i}$ and principal axes $\boldsymbol{u}_{i}$ [2].

Furthermore, the potential energy function of an ideal stiffness depends only on the relative pose of the two attached bodies and is port symmetric [2]. ${ }^{2}$ Considering the displacement $\underline{\boldsymbol{y}}_{d}^{r} \triangleq \log \underline{\boldsymbol{x}}_{d}^{r}$, with $\underline{\boldsymbol{x}}_{d}^{r} \triangleq \underline{\boldsymbol{x}}_{r}^{*} \underline{\boldsymbol{x}}_{d}$, the positive definite matrices $\overline{\boldsymbol{K}}_{\phi} \in \mathbb{R}^{3 \times 3}$ and $\boldsymbol{K}_{p} \in \mathbb{R}^{3 \times 3}$ that represent the rotational and translational stiffness matrices, respectively, and that there

\footnotetext{
${ }^{2}$ Port symmetry implies that the potential energy is the same whether seen from $\mathcal{F}_{r}$ or $\mathcal{F}_{d}$.
}

is no coupling between translation and rotation, the elastic potential energy is given by

$$
\mathcal{U}=\left(\operatorname{vec}_{6} \underline{\boldsymbol{y}}_{d}^{r}\right)^{T} \boldsymbol{K}_{d} \operatorname{vec}_{6} \underline{\boldsymbol{y}}_{d}^{r},
$$

with $\boldsymbol{K}_{d}=2 \operatorname{diag}\left(\boldsymbol{K}_{\phi}, \boldsymbol{K}_{p}^{\prime}\right)$, where ${ }^{3}$

$$
\boldsymbol{K}_{p}^{\prime} \triangleq \frac{1}{2} \boldsymbol{K}_{p}+\frac{1}{2} \boldsymbol{R}_{d}^{r} \boldsymbol{K}_{p} \boldsymbol{R}_{d}^{r T},
$$

with $\boldsymbol{R}_{d}^{r}=\overline{\boldsymbol{I}} \stackrel{+}{H}_{4}\left(\boldsymbol{r}_{d}^{r}\right) \overline{\boldsymbol{H}}_{4}\left(\boldsymbol{r}_{d}^{r *}\right) \overline{\boldsymbol{I}}^{T}$ being the rotation matrix from frame $\mathcal{F}_{r}$ to $\mathcal{F}_{d}$, which guarantees that the potential energy is port symmetric.

Deriving (7), the power is given by ${ }^{4}$

$$
\begin{aligned}
\dot{\mathcal{U}}=2 & \left(\operatorname{vec}_{6} \underline{\dot{\boldsymbol{y}}}_{d}^{r}\right)^{T} \boldsymbol{K}_{d} \operatorname{vec}_{6} \underline{\boldsymbol{y}}_{d}^{r} \\
& +2\left(\operatorname{vec}_{6} \underline{\boldsymbol{y}}_{d}^{r}\right)^{T}\left[\begin{array}{cc}
\mathbf{0}_{3 \times 3} & \mathbf{0}_{3 \times 3} \\
\mathbf{0}_{3 \times 3} & \dot{\boldsymbol{R}}_{d}^{r} \boldsymbol{K}_{p} \boldsymbol{R}_{d}^{r T}
\end{array}\right] \operatorname{vec}_{6} \underline{\boldsymbol{y}}_{d}^{r} .
\end{aligned}
$$

Using (2) in (9) and the fact that $\dot{\boldsymbol{R}}_{d}^{r}=\boldsymbol{S}\left(\operatorname{vec}_{3} \boldsymbol{\omega}_{r d}^{r}\right) \boldsymbol{R}_{d}^{r}$ [13], we obtain

$$
\begin{aligned}
\dot{\mathcal{U}}=\left(\operatorname{vec}_{6} \underline{\boldsymbol{\zeta}}_{r d}^{r}\right)^{T}\left(\boldsymbol{E}^{-T}\right. & \left(\underline{\boldsymbol{x}}_{d}^{r}\right) 2 \boldsymbol{K}_{d} \\
& \left.+\left[\begin{array}{cc}
\mathbf{0}_{3 \times 3} & \boldsymbol{K}_{p}^{\prime \prime} \\
\mathbf{0}_{3 \times 3} & \mathbf{0}_{3 \times 3}
\end{array}\right]\right) \operatorname{vec}_{6} \underline{\boldsymbol{y}}_{d}^{r} .
\end{aligned}
$$

with $\boldsymbol{K}_{p}^{\prime \prime}=\boldsymbol{S}^{T}\left(\operatorname{vec}_{3} \boldsymbol{p}_{r d}^{r}\right) \boldsymbol{R}_{d}^{r} \boldsymbol{K}_{p} \boldsymbol{R}_{d}^{r T}$. Substituting (4) in (10) yields

$$
\dot{\mathcal{U}}=\left(\operatorname{vec}_{6} \underline{\boldsymbol{\zeta}}_{r d}^{r}\right)^{T} \boldsymbol{I}^{\#} \operatorname{vec}_{6} \underline{\boldsymbol{\varsigma}}_{E}^{r},
$$

where $\operatorname{vec}_{6} \underline{\boldsymbol{\varsigma}}_{E}^{r} \triangleq \boldsymbol{I}^{\#} \boldsymbol{K}_{d}^{\prime} \operatorname{vec}_{6} \underline{\boldsymbol{y}}_{d}^{r}$ is the elastic wrench with respect to $\mathcal{F}_{r}$, with

$$
\boldsymbol{K}_{d}^{\prime}=\left[\begin{array}{cc}
\boldsymbol{K}_{\phi}^{\prime} & \boldsymbol{K}_{p}^{\prime \prime} \\
\mathbf{0}_{3 \times 3} & 2 \boldsymbol{K}_{p}^{\prime}
\end{array}\right]
$$

where

$$
\boldsymbol{K}_{\phi}^{\prime}=2 \overline{\boldsymbol{I}} \overline{\boldsymbol{H}}_{4}\left(\boldsymbol{r}_{d}^{r *}\right) \boldsymbol{Q}_{4}^{+T}\left(\boldsymbol{r}_{d}^{r}\right) \boldsymbol{K}_{\phi} .
$$

Hence, the impedance equation is given by $\operatorname{vec}_{6} \underline{\boldsymbol{\varsigma}}_{I}^{r}+\operatorname{vec}_{6} \underline{\boldsymbol{\varsigma}}_{D}^{r}+\operatorname{vec}_{6} \underline{\boldsymbol{\varsigma}}_{E}^{r}=-\operatorname{vec}_{6} \underline{\boldsymbol{\varsigma}}^{r}, \quad$ which leads to

$$
\boldsymbol{M}_{d} \dot{\boldsymbol{\zeta}}_{r d}^{r}+\boldsymbol{B}_{d} \boldsymbol{\zeta}_{r d}^{r}+\boldsymbol{K}_{d}^{\prime} \boldsymbol{y}_{d}^{r}=-\boldsymbol{I}^{\#} \boldsymbol{\varsigma}^{r},
$$

where $\underline{\varsigma}^{r}=\boldsymbol{r}_{r}^{*} \underline{\underline{ }} \boldsymbol{r}_{r}$ is the external wrench acting on the robot end-effector expressed in relation to $\mathcal{F}_{r}$. Also, $\boldsymbol{\zeta}_{r d}^{r} \triangleq \operatorname{vec}_{6} \underline{\boldsymbol{\zeta}}_{r d}^{r}$, $\boldsymbol{y}_{d}^{r} \triangleq \operatorname{vec}_{6} \underline{\boldsymbol{y}}_{d}^{r}$, and $\boldsymbol{\varsigma}^{r}=\operatorname{vec}_{6} \underline{\boldsymbol{\varsigma}}^{r}$.

The dual of the impedance is the admittance equation [14], which is given by

$$
\dot{\boldsymbol{\zeta}}_{r d}^{r}=\boldsymbol{M}_{d}^{-1}\left(-\boldsymbol{I}^{\#} \boldsymbol{\varsigma}^{r}-\boldsymbol{B}_{d} \boldsymbol{\zeta}_{r d}^{r}-\boldsymbol{K}_{d}^{\prime} \boldsymbol{y}_{d}^{r}\right) .
$$

Remark 2. The orientation part of the stiffness term in (14) and (15) (i.e., $\boldsymbol{K}_{\phi}^{\prime} \operatorname{vec}_{3}\left(\boldsymbol{n}_{r d}^{r} \phi / 2\right)$ ) is linear with respect to the

\footnotetext{
${ }^{3}$ This transformation is only needed for translation because $\log \boldsymbol{r}_{d}^{r}=$ $-\log \boldsymbol{r}_{r}^{d}$, but $\log \underline{\boldsymbol{x}}_{d}^{r} \neq-\log \underline{\boldsymbol{x}}_{r}^{d}$.

${ }^{4}$ It can be verified by direct calculation that $\dot{\boldsymbol{R}}_{d}^{r} \boldsymbol{K}_{p} \boldsymbol{R}_{d}^{r T}$ is a symmetric matrix.
} 
angle $\phi$. As a result, the closed-loop error decays exponentially, which is not true for other controllers in the literature, as better discussed in Section IV-E.

Lemma 3. The matrix $\boldsymbol{K}_{d}^{\prime}$ in (12) is invertible.

Proof: To prove that $\boldsymbol{K}_{d}^{\prime}$ is invertible, it is sufficient to prove that $\boldsymbol{K}_{\phi}^{\prime}$ (13) and $\boldsymbol{K}_{p}^{\prime}$ (8) are full rank. Since $\boldsymbol{K}_{p}$ is positive definite, then $\boldsymbol{R}_{d}^{r} \boldsymbol{K}_{p} \boldsymbol{R}_{d}^{r T}$ is also positive definite. Also, the sum of two positive-definite matrices is also positivedefinite, therefore $\boldsymbol{K}_{p}^{\prime}$ is positive definite and thus has full rank [12]. By (13), $\boldsymbol{K}_{\phi}^{\prime}=4 \boldsymbol{W}^{-T} \boldsymbol{K}_{\phi}$ and since $\boldsymbol{W}$ and $\boldsymbol{K}_{\phi}$ are full rank, so it is $\boldsymbol{K}_{\phi}^{\prime}$ [11]. Hence, (12) is full rank and thus invertible.

Theorem 4. Assuming that the inner motion controller accurately tracks the trajectory generated by the admittance controller in the outer loop-i.e., the inner loop dynamics is not taken into account-the closed-loop system given by (14) is passive, hence stable. Moreover, when in free-motion, the only equilibrium point is given by $\underline{\boldsymbol{y}}_{d}^{r}=0$, which implies that $\underline{\boldsymbol{x}}_{r}=\underline{\boldsymbol{x}}_{d}$.

Proof: Given the Hamiltonian $\mathcal{E}=\mathcal{K}+\mathcal{U}$, we use (6), (11), and (14) to obtain

$$
\dot{\mathcal{E}}=\dot{\mathcal{K}}+\dot{\mathcal{U}}=-\left(\boldsymbol{\zeta}_{r d}^{r}\right)^{T} \boldsymbol{I}^{\#} \boldsymbol{\varsigma}^{r}-\left(\boldsymbol{\zeta}_{r d}^{r}\right)^{T} \boldsymbol{B}_{d} \boldsymbol{\zeta}_{r d}^{r}
$$

where $\mathcal{E}$ is the stored energy and thus positive, $-\boldsymbol{I}^{\#} \boldsymbol{\varsigma}^{r}$ is the system input, $\boldsymbol{\zeta}_{r d}^{r}$ is the output, and $\boldsymbol{\zeta}_{r d}^{r T} \boldsymbol{B}_{d} \boldsymbol{\zeta}_{r d}^{r} \geq 0$. Therefore, the system represents a passive mapping from $-\boldsymbol{I}^{\#} \boldsymbol{\varsigma}^{r}$ to $\boldsymbol{\zeta}_{r d}^{r}$ and is, hence, stable [15]. Moreover, it is dissipative, with the dissipative power given by $\boldsymbol{\zeta}_{r d}^{r T} \boldsymbol{B}_{d} \boldsymbol{\zeta}_{r d}^{r}$.

Furthermore, in the case of free motion (i.e., $\boldsymbol{\varsigma}^{r}=\mathbf{0}$ ), $\dot{\mathcal{E}}=0$ if and only if $\boldsymbol{\zeta}_{r d}^{r}=\mathbf{0}$. Also, because the system is dissipative, if $\varsigma^{r}=\mathbf{0}$ and $\boldsymbol{\zeta}_{r d}^{r}=\mathbf{0}$, then $\dot{\zeta}_{r d}^{r}=\mathbf{0}$. Therefore, from (14), we have

$$
\boldsymbol{K}_{d}^{\prime} \boldsymbol{y}_{d}^{r}=\mathbf{0}
$$

which implies that $\underline{\boldsymbol{y}}_{d}^{r}=0$ is the only equilibrium point since by Lemma $3 \boldsymbol{K}_{d}^{\prime}$ is invertible. Moreover, $\underline{\boldsymbol{y}}_{d}^{r}=0 \Longrightarrow \underline{\boldsymbol{x}}_{d}^{r}=1 \Longrightarrow \underline{\boldsymbol{x}}_{r}=\underline{\boldsymbol{x}}_{d}$.

1) Unwinding problem: Although the only equilibrium point is $\underline{\boldsymbol{y}}_{d}^{r}=0 \Longrightarrow \underline{\boldsymbol{x}}_{d}^{r}=1$, both $\underline{\boldsymbol{x}}_{d}^{r}=1$ and $\underline{\boldsymbol{x}}_{d}^{r}=-1$ represent the same pose [6]. Considering the control laws (14) and (15), if $\underline{\boldsymbol{x}}_{d}^{r}=-1$ the robot will move to reach $\underline{\boldsymbol{x}}_{d}^{r}=1$, which is undesirable (this unnecessary motion is called unwinding). To prevent this situation, we propose a new definition of $\underline{\boldsymbol{y}}_{d}^{r}$ to be used in the impedance/admittance equation; that is,

$$
\underline{\boldsymbol{y}}_{d}^{r} \triangleq \begin{cases}\log \underline{\boldsymbol{x}}_{d}^{r}, & \text { if }\left\|\underline{\boldsymbol{x}}_{d}^{r}-1\right\|_{2} \leq\left\|\underline{\boldsymbol{x}}_{d}^{r}+1\right\|_{2}, \\ \log \left(-\underline{\boldsymbol{x}}_{d}^{r}\right), & \text { otherwise. }\end{cases}
$$

Therefore, $\underline{\boldsymbol{y}}_{d}^{r}$ will be zero when $\underline{\boldsymbol{x}}_{d}^{r}=1$ and $\underline{\boldsymbol{x}}_{d}^{r}=-1$, and the closed-loop system trajectories will always choose the smallest spatial distance to the stable points 1 and -1 , thus preventing the unwinding problem.

Theorem 5. The system given by (14) where $\boldsymbol{y}_{d}^{r}=\operatorname{vec}_{6} \underline{\boldsymbol{y}}_{d}^{r}$, with $\underline{\boldsymbol{y}}_{d}^{r}$ defined as in (17) is stable. Furthermore, it has two stable equilibrium points: $\underline{\boldsymbol{x}}_{d}^{r}$ and $-\underline{\boldsymbol{x}}_{d}^{r}$.
Proof: Considering $\underline{\boldsymbol{y}}_{d}^{r}=\log \underline{\boldsymbol{a}}$, by Theorem 4 the system is in equilibrium when $\underline{\boldsymbol{a}}=1$. Since according to (17) $\underline{\boldsymbol{a}} \in \underline{\mathcal{S}}$ can be $\underline{\boldsymbol{x}}_{d}^{r}$ or $-\underline{\boldsymbol{x}}_{d}^{r}$, this means that we have two equilibrium points, $\underline{\boldsymbol{x}}_{d}^{r}$ and $-\underline{\boldsymbol{x}}_{d}^{r}$, both stable.

\section{B. Inner-loop controller}

Since a large class of robots is actuated in velocity, we use a kinematic controller in the inner loop to track a desired end-effector trajectory while controlling the desired apparent impedance by means of the admittance controller in the outer loop. Using the relations (1) and (2) and their derivatives, the reference $\dot{\boldsymbol{\zeta}}_{r d}^{r}$ from (15) is integrated and transformed into the reference trajectory given by $\left\{\underline{\boldsymbol{x}}_{r}(t), \underline{\boldsymbol{x}}_{r}(t), \underline{\ddot{\boldsymbol{x}}}_{r}(t)\right\}$.

To track the trajectory, we first consider the error $\underline{\tilde{x}}=\underline{\boldsymbol{x}}^{*} \underline{\boldsymbol{x}}_{r}$, where $\underline{\boldsymbol{x}}$ is the current end-effector pose, which is calculated by using the forward kinematics (we assume a perfect model), and $\underline{\boldsymbol{x}}_{r}$ is the reference pose. Thus,

$$
\operatorname{vec}_{8} \underline{\dot{\tilde{\boldsymbol{x}}}}=\overline{\boldsymbol{H}}_{8}\left(\underline{\boldsymbol{x}}_{r}\right) \boldsymbol{C}_{8} \operatorname{vec}_{8} \underline{\dot{\boldsymbol{x}}}+\overline{\boldsymbol{H}}_{8}\left(\underline{\dot{\boldsymbol{x}}}_{r}\right) \boldsymbol{C}_{8} \operatorname{vec}_{8} \underline{\boldsymbol{x}},
$$

where $\overline{\boldsymbol{H}}_{8}(\cdot) \in \mathbb{R}^{8 \times 8}$ satisfies $\operatorname{vec}_{8}(\underline{\boldsymbol{a} \boldsymbol{b}})=\overline{\boldsymbol{H}}_{8}(\underline{\boldsymbol{b}}) \operatorname{vec}_{8} \underline{\boldsymbol{a}}$ and $\boldsymbol{C}_{8}=\operatorname{diag}(1,-1,-1,-1,1,-1,-1,-1)$ [7].

Second, we assume that the forward kinematics model $\underline{\boldsymbol{x}}=\underline{\boldsymbol{f}}(\boldsymbol{q})$, where $\boldsymbol{q} \in \mathbb{R}^{n}$ is the robot configuration and $\underline{f}: \mathbb{R}^{n} \rightarrow \underline{\mathcal{S}}$, and the differential forward kinematics $\overline{\operatorname{vec}}_{8} \underline{\dot{\boldsymbol{x}}}=\boldsymbol{J}(\boldsymbol{q}) \dot{\boldsymbol{q}}$, with $\boldsymbol{J}(\boldsymbol{q}) \in \mathbb{R}^{8 \times n}$ being the Jacobian matrix, are available [8].

Finally, considering (1), the time derivative of (18), and defining the desired closed-loop task error dynamics as

$$
\boldsymbol{a}_{y} \triangleq \operatorname{vec}_{8} \underline{\ddot{\tilde{\boldsymbol{y}}}}=-\boldsymbol{K}_{D} \operatorname{vec}_{8} \underline{\dot{\tilde{\boldsymbol{y}}}}-\boldsymbol{K}_{P} \operatorname{vec}_{8} \underline{\tilde{\boldsymbol{y}}},
$$

with $\mathbb{R}^{6 \times 6} \ni \boldsymbol{K}_{D}, \boldsymbol{K}_{P}>0$, the control law is given by

$$
\boldsymbol{u}_{\mathrm{nom}} \triangleq \ddot{\boldsymbol{q}}=\boldsymbol{N}^{+}\left(\boldsymbol{Q}_{8}(\underline{\tilde{\boldsymbol{x}}}) \boldsymbol{a}_{y}+\boldsymbol{z}\right),
$$

where $\boldsymbol{z} \triangleq \dot{\boldsymbol{Q}}_{8}(\underline{\tilde{\boldsymbol{x}}}) \operatorname{vec}_{6} \underline{\dot{\tilde{\boldsymbol{y}}}}-\dot{\boldsymbol{A}} \operatorname{vec}_{8} \underline{\boldsymbol{x}}-\boldsymbol{A} \boldsymbol{J}(\boldsymbol{q}) \dot{\boldsymbol{q}}-\dot{\boldsymbol{N}} \dot{\boldsymbol{q}}, \underline{\tilde{\boldsymbol{y}}} \triangleq$ $\log \underline{\tilde{\boldsymbol{x}}}, \boldsymbol{N} \triangleq \overline{\boldsymbol{H}}_{8}\left(\underline{\boldsymbol{x}}_{d}\right) \boldsymbol{C}_{8} \boldsymbol{J}(\boldsymbol{q})$, and $\boldsymbol{A} \triangleq \overline{\boldsymbol{H}}_{8}\left(\underline{\dot{\boldsymbol{x}}}_{d}\right) \boldsymbol{C}_{8}$.

Theorem 6. The closed-loop error dynamics of a system modeled as $\operatorname{vec}_{8} \underline{\ddot{\boldsymbol{x}}}=\dot{\boldsymbol{J}}(\boldsymbol{q}) \dot{\boldsymbol{q}}+\boldsymbol{J}(\boldsymbol{q}) \ddot{\boldsymbol{q}}$ under the control law (20) is given by

$$
\operatorname{vec}_{6} \underline{\ddot{\tilde{\boldsymbol{y}}}}+\boldsymbol{K}_{D} \operatorname{vec}_{6} \underline{\dot{\tilde{\boldsymbol{y}}}}+\boldsymbol{K}_{P} \operatorname{vec}_{6} \underline{\tilde{\boldsymbol{y}}}=\mathbf{0}
$$

and is asymptotically stable in the Lyapunov sense when $\boldsymbol{K}_{P}, \boldsymbol{K}_{D}>0$.

Proof: Taking the time-derivative of (18) together with $\operatorname{vec}_{8} \underline{\ddot{\boldsymbol{x}}}=\dot{\boldsymbol{J}}(\boldsymbol{q}) \dot{\boldsymbol{q}}+\boldsymbol{J}(\boldsymbol{q}) \ddot{\boldsymbol{q}}$ yields

$$
\begin{aligned}
\operatorname{vec}_{8} \underline{\ddot{\tilde{\boldsymbol{x}}}}=\overline{\boldsymbol{H}}_{8}\left(\underline{\boldsymbol{x}}_{d}\right) \boldsymbol{C}_{8}(\dot{\boldsymbol{J}}(\boldsymbol{q}) \dot{\boldsymbol{q}}+\boldsymbol{J}(\boldsymbol{q}) \ddot{\boldsymbol{q}}) \\
+2 \boldsymbol{A J}(\boldsymbol{q}) \dot{\boldsymbol{q}}+\overline{\boldsymbol{H}}_{8}\left(\underline{\ddot{\boldsymbol{x}}}_{d}\right) \boldsymbol{C}_{8} \operatorname{vec}_{8} \underline{\boldsymbol{x}} .
\end{aligned}
$$

Since the time derivative of (1) is $\operatorname{vec}_{8} \underline{\ddot{\tilde{x}}}=\dot{Q}_{8}(\underline{\tilde{x}}) \operatorname{vec}_{6} \underline{\dot{\tilde{y}}}+$ $\boldsymbol{Q}_{8}(\underline{\tilde{\boldsymbol{x}}}) \operatorname{vec}_{6} \underline{\tilde{\tilde{\boldsymbol{y}}}}$, we replace it in (22) and isolate $\boldsymbol{N} \ddot{\boldsymbol{q}}$ to obtain

$$
\boldsymbol{N} \ddot{\boldsymbol{q}}=\boldsymbol{z}+\boldsymbol{Q}_{8}(\underline{\tilde{\boldsymbol{x}}}) \operatorname{vec}_{6} \underline{\ddot{\tilde{\boldsymbol{y}}}}
$$


which implies that $\boldsymbol{z}$ and $\boldsymbol{Q}_{8}(\underline{\tilde{\boldsymbol{x}}}) \operatorname{vec}_{6} \ddot{\tilde{\boldsymbol{y}}}$ are in the range space of $N .^{5}$ Therefore, replacing $(20)$ in (23) yields $\boldsymbol{N} \boldsymbol{N}^{+} \boldsymbol{Q}_{8}(\underline{\tilde{x}}) \boldsymbol{a}_{y}+\boldsymbol{N} \boldsymbol{N}^{+} \boldsymbol{z}=\boldsymbol{z}+\boldsymbol{Q}_{8}(\underline{\tilde{\boldsymbol{x}}}) \operatorname{vec}_{6} \ddot{\tilde{\boldsymbol{y}}}$. Thus, $\boldsymbol{Q}_{8}(\underline{\tilde{\boldsymbol{x}}}) \boldsymbol{a}_{y}=\boldsymbol{Q}_{8}(\underline{\tilde{\tilde{x}}}) \operatorname{vec}_{6} \underline{\ddot{\tilde{y}}}$, which implies $\boldsymbol{a}_{y}=\operatorname{vec}_{6} \underline{\ddot{\tilde{y}}}$ because $\boldsymbol{Q}_{8}(\underline{\tilde{x}})$ is full-column rank [10]. Using (19), we obtain the closed-loop error dynamics given by (21), which is asymptotically stable in the Lyapunov sense for the PIS $\left[\left(\operatorname{vec}_{6} \underline{\tilde{\boldsymbol{y}}}\right)^{T}\left(\operatorname{vec}_{6} \underline{\dot{\boldsymbol{y}}}\right)^{T}\right]^{T}=\mathbf{0} \in \mathbb{R}^{12}$ provided $\boldsymbol{K}_{P}, \boldsymbol{K}_{D}>$ 0 [16, pp. 229-230].

In case of redundant robots, the joint velocities can be different from zero even if the system is in equilibrium. To prevent that situation, we add a dissipative term as $\boldsymbol{u}_{\mathrm{dis}}=k_{\mathrm{dis}}\left(\mathbf{1}_{n}\left\|\operatorname{vec}_{6} \underline{\dot{\boldsymbol{y}}}\right\|-\dot{\boldsymbol{q}}\right)$, where $\mathbf{1}_{n}$ is an $n$-dimensional column vector of ones and $k_{\mathrm{dis}} \in(0, \infty)$ [17]. Thus, the control law becomes

$$
\boldsymbol{u}=\boldsymbol{u}_{\mathrm{nom}}+\boldsymbol{u}_{\mathrm{dis}} .
$$

Remark 7. The solution (17) to the unwinding problem can also be applied to the kinematic controller (24) by using $\tilde{\boldsymbol{y}}$ and $\underline{\tilde{\boldsymbol{x}}}$ instead of $\underline{\boldsymbol{y}}_{d}^{r}$ and $\underline{\boldsymbol{x}}_{d}^{r}$, respectively.

\section{Simulation AND EXPERIMENTAL RESUlts ${ }^{6}$}

To evaluate our proposed control architecture, simulations were run in MATLAB using the DQ Robotics library [18]. Experiments were run on a KUKA LWR4+ robot manipulator, equipped with a computer with two Intel Xeon $2.4 \mathrm{Ghz}$ hexacore processors with $32 \mathrm{~Gb}$ of RAM each, and a 64-bit Anarchy Linux version 1.4 (Linux 4.19.50-rt22-2-rt-lts) using the C++ version of DQ Robotics. The robot is equipped with one ATI Mini 45 force/torque sensor at its end-effector, and it only reacts to wrenches applied at the end-effector. ${ }^{7}$

Since the KUKA LWR4+ is actuated in position, the control input (24) is numerically integrated twice, using Newton's first-order approximation, to obtain velocity $(\dot{\boldsymbol{q}})$ and position signals $(\boldsymbol{q})$. To prevent reaching the joints maximum velocities, they were saturated in $0.2 \mathrm{rad} / \mathrm{s}$.

Both simulations and experiments were run with a sampling time of $5 \mathrm{~ms}$. The matrices in (15) were chosen as $\boldsymbol{M}_{d}=1.5 \boldsymbol{I}_{6 \times 6}, \boldsymbol{B}_{d}=300 \boldsymbol{I}_{6 \times 6}, \boldsymbol{K}_{p}=80 \boldsymbol{I}_{3 \times 3}$, and $\boldsymbol{K}_{\phi}=80 \boldsymbol{I}_{3 \times 3}$, whereas in (24) they were chosen as $\boldsymbol{K}_{P}=25 \boldsymbol{I}_{6 \times 6}, \boldsymbol{K}_{D}=10 \boldsymbol{I}_{6 \times 6}$, and $k_{\mathrm{dis}}=1$. In the kinematic controller, the pose $\underline{x}(\boldsymbol{q})$ is given by the forward kinematics, which is obtained by using dual quaternion algebra [8].

\section{A. Simulation of unwinding}

We performed a simulation of a free-flying rigid body to show that, when using (17), the end-effector follows the smallest path towards the desired pose, whereas without considering

\footnotetext{
${ }^{5}$ Given $\boldsymbol{s} \in \mathbb{R}^{n}$ and $\boldsymbol{G} \in \mathbb{R}^{n \times m}$, if $\boldsymbol{s} \in$ range $\boldsymbol{G}$ then $\boldsymbol{G} \boldsymbol{G}^{+} \boldsymbol{s}=\boldsymbol{s}$. [11]

${ }^{6}$ See the accompanying video and its extended version at https://youtu.be/ SMXQC6B6DTg.

${ }^{7}$ If the robot is equipped with torque sensors at the joints, the joint torques $\tau$ may be projected onto the end-effector by using the well-known relationship $\boldsymbol{\tau}=\boldsymbol{J}_{G}^{T}(\boldsymbol{q}) \operatorname{vec}_{6} \underline{\boldsymbol{\varsigma}}$, where $\boldsymbol{J}_{G}$ is the geometric Jacobian. Hence, the admittance controller can be used to encompass compliant motions with distributed contacts [19].
}

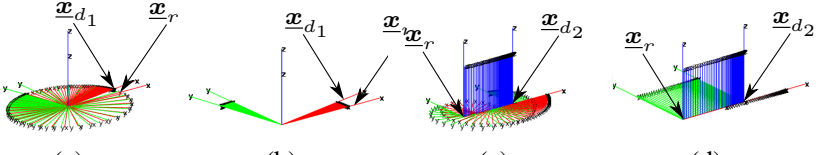

(a)

(b)

(c)

(d)

Figure 2: Simulation of a free-flying rigid body under the unwinding phenomenon ( $a$ and $c$ ), and path using solution (17) $(b$ and $d)$.

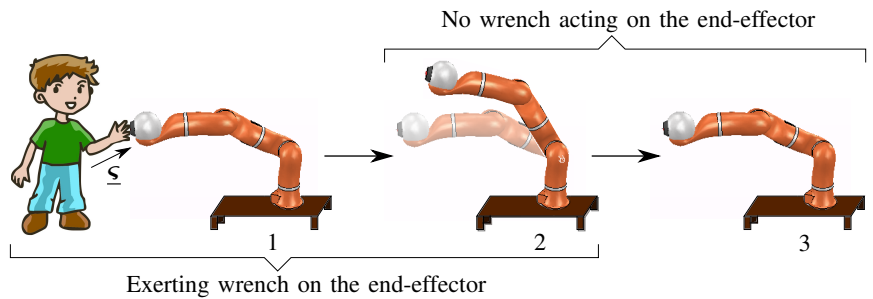

Figure 3: The experiments are divided in two parts: from 1 to 2 , where a wrench is applied to the end-effector, which makes $\underline{\boldsymbol{x}}_{r}$ be different from a constant $\underline{\boldsymbol{x}}_{d}$; and from 2 to 3 , where the controllers are applied in free-motion, such that $\underline{\boldsymbol{x}}_{r}$ returns to $\underline{\boldsymbol{x}}_{d}$.

(17), the end-effector performs an unnecessary rotation. Fig. 2 shows the simulation for two different initial displacements between $\underline{\boldsymbol{x}}_{r}$ and $\underline{\boldsymbol{x}}_{d}$, namely $\underline{\boldsymbol{x}}_{d_{1}}^{r}$ and $\underline{\boldsymbol{x}}_{d_{2}}^{r}$. In the first case, (Figs. 2a-2b), $\underline{\boldsymbol{x}}_{d_{1}}^{r}=\cos (\pi+0.1)+\hat{k} \sin (\pi+0.1)$ is closer to -1 than to 1 . Thus, without using (17), the body executes a rotation of almost $2 \pi$ to reach the desired pose (Fig. 2a). When considering (17), the rotation is much smaller (Fig. 2b). In the second case (Figs. 2c-2d), $\underline{\boldsymbol{x}}_{d_{2}}^{r}=-1-\varepsilon(1 / 2) 0.3 \hat{\imath}$ is closer to -1 than to 1 and consists of a pure translation. In this case, without the solution for the unwinding problem, the body executes a rotation of $2 \pi$, whereas with the solution it translates while keeping its orientation.

\section{B. Experimental setup}

Consider $\underline{\boldsymbol{x}}_{r}(0)=\underline{\boldsymbol{x}}_{d}(0) \triangleq \underline{\boldsymbol{x}}_{0}$. When an external contact wrench acts on the end-effector, the reference pose $\underline{\boldsymbol{x}}_{r}$ becomes different from $\underline{x}_{d}$ to ensure the desired apparent impedance, and the end-effector follows the trajectory given by $\underline{\boldsymbol{x}}_{r}(t)$. This situation is illustrated in the movement from 1 to 2 in Fig. 3. When the contact is released, $\underline{\boldsymbol{x}}_{r}$ (and consequently $\underline{x}$ ) returns to the desired pose $\underline{x}_{d}$, thanks to the dynamics determined by the admittance controller, as illustrated by movement from 2 to 3 in Fig. 3.

Two different experiments were performed: first, an external wrench acts on the end-effector (first part of Fig. 3); second, the robot performs a free motion (second part of Fig. 3).

The following analyses were made to compare our proposed controller (15) with one of the main admittance controllers in the state of art, given by (25), named here as ACIm (see Appendix A). The inner-loop controller was the same for both cases and both admittance controllers.

\section{Experiments with an external wrench}

To apply a wrench at the end-effector, a person pushes the manipulator, which moves complacently. The translation and orientation of the current end-effector pose $\underline{x}$, the reference pose $\underline{\boldsymbol{x}}_{r}$, and the desired pose $\underline{\boldsymbol{x}}_{d}$ are shown in Fig. 4, for 


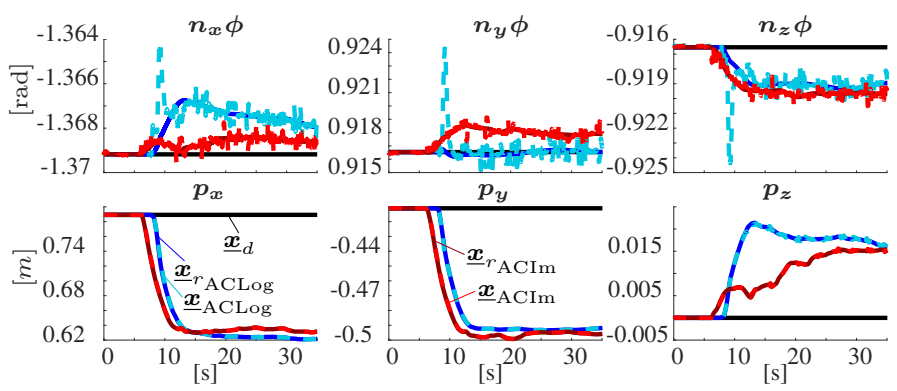

Figure 4: Results for the experiment in which a contact wrench acts on the robot end-effector. The figure shows the position and orientation of current, reference, and desired poses.

both controllers. Being exerted by a human, the wrench is not exactly the same for the two controllers. However, both controllers generated a reference trajectory $\underline{\boldsymbol{x}}_{r}$ different from $\underline{\boldsymbol{x}}_{d}$, as expected, to ensure a compliant behavior according to the desired impedance, and this trajectory was followed by the end-effector, thanks to the inner-loop controller. Moreover, the results showed a control signal $\boldsymbol{u}$ with similar magnitude for both controllers.

\section{Experiments in free-motion}

Since the experiment of human-robot interaction with a contact wrench is limited for comparison, as it is more susceptible to variations in the interaction wrench, deeper analyses were made in the second part of the movement, when there is no wrench acting on the end-effector. As a way to see if there is a significant difference between the performance of the two controllers ((15) and (25)), statistical analyses were performed considering

1) the difference between the desired error dynamics and the experimental one;

2) the control effort, maximum and mean values of the control signal, regarding the admittance controller;

3) the control effort, maximum and mean values of the control signal, regarding the kinematic controller.

Given an initial pose, different desired poses are generated randomly and each generated pose is the same for each controller to allow for a fair comparison.

1) Statistical methodology: We used the Wilcoxon Rank Sum Test [20], which is a nonparametric test used to check whether two independent samples are from populations with the same distribution. Also, we use the following concepts:

1) p-value, which is the lowest significance level that would lead to the rejection of the null-hypothesis. This occurs if and only if the p-value is smaller than the significance level $\alpha$, which is the probability of occurrence of a false positive;

2) power of the test, which is given by $(1-\beta)$, where $\beta$ is the probability of occurrence of a false negative;

3) minimally interesting effect, which is the smallest difference between the controllers we are interested in detecting, regarding each one of the aspects 1,2 , and 3.

2) Estimation of the appropriate number of samples: Each controller was initially run 30 times to determine the necessary
Table I: Minimally interesting effect, for each comparison.

\begin{tabular}{|c|c|c|c|c|c|}
\hline \multicolumn{2}{|c|}{ Error discrepancy } & \multicolumn{2}{|c|}{$\begin{array}{l}\text { Control signal of outer } \\
\text { loop }\end{array}$} & \multicolumn{2}{|c|}{$\begin{array}{l}\text { Control signal of inner } \\
\text { loop }\end{array}$} \\
\hline$\overline{\delta \int \tilde{e}}$ & 20 & $\delta_{\int\left\|\ddot{\boldsymbol{x}}_{r}\right\|}$ & 10 & $\delta_{\int\|\boldsymbol{u}\|}$ & 25 \\
\hline$\delta \mu_{\tilde{e}}$ & 0.01 & $\delta_{\mu_{\left\|\ddot{\boldsymbol{x}}_{r}\right\|}}$ & 0.01 & $\delta_{\mu\|\boldsymbol{u}\|}$ & 0.1 \\
\hline$\delta_{\max (\tilde{\boldsymbol{e}})}$ & 0.02 & $\delta_{\max \left(\left\|\ddot{\boldsymbol{x}}_{r}\right\|\right)}$ & 10 & $\delta_{\max (\|\boldsymbol{u}\|)}$ & 25 \\
\hline
\end{tabular}

number os samples based on the variance of the data. To generate these 30 pairs of initial/final end-effector poses, one initial robot configuration $\boldsymbol{q}_{0}$ was arbitrarily chosen and 30 different configurations were generated by a normal distribution $\mathcal{N}\left(\boldsymbol{q}_{0}, 0.5\right)$, and the end-effector poses were calculated by using the forward kinematics. We calculated the variance for each run, and chose a significance level of $\alpha=0.05$, a power of 0.85 , and a minimally interesting effect as shown in Table I. With those parameters and the variance, the number of samples was calculated using the two-sample t-test power calculation available in $\mathrm{R}$, resulting in a value lower than 30 for all cases. Hence, the analyses were made with the 30 samples already collected.

3) Statistical analyses of the error dynamics: We first analyze the difference between the desired and the actual error norm decay. More specifically, the error is given by

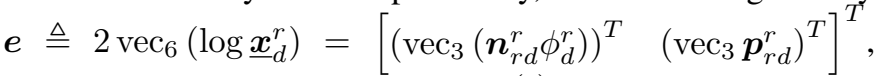
and the desired error dynamics $\boldsymbol{e}_{d}(t)$ is given by the solution of the equation $\boldsymbol{M}_{d} \ddot{\boldsymbol{e}}+\boldsymbol{B}_{d} \dot{\boldsymbol{e}}+\boldsymbol{K}_{d} \boldsymbol{e}=\mathbf{0}$, with $\boldsymbol{K}_{d}=$ $\operatorname{diag}\left(\boldsymbol{K}_{\phi}, \boldsymbol{K}_{p}\right)$. Therefore, given a discrepancy function defined as $\tilde{\boldsymbol{e}}(t) \triangleq\|\| \boldsymbol{e}_{d}(t)\|-\| \boldsymbol{e}(t)\|\|,{ }^{8}$ the first analysis concerns the total discrepancy, given by $\sqrt{\int_{0}^{T} \tilde{e}(t)^{2} d t}$, for $T=35 \mathrm{~s}$, along the trajectory. Fig. 5 shows that the total discrepancy is very small for the ACLog, but this difference presents larger values for the ACIm. The same is observed for the maximum value of the discrepancy function (Fig. 5a) and its mean value $\mu_{\tilde{\boldsymbol{e}}(t)}$ (Fig. 5a). Moreover, the ACIm presents outliers in all three cases, indicating a even larger discrepancy for some cases. The p-values for the Wilcoxon Rank Sum Test were respectively $1.675 \times 10^{-12}, 2.753 \times^{-9}$, and $5.998 \times 10 e^{-13}$, all smaller than the significance level $\alpha=0.05$, therefore the null hypothesis that the population are of the same type is rejected. By the box-plot and the Wilcoxon Rank Sum Test, we conclude that the ACLog obtained a better performance. The larger error discrepancy of the ACIm over the ACLog may be partially explained by the non-linearity of the former, as described in Section IV-E. Nonetheless, this difference may not be critical for general applications.

4) Statistical analyses of the outer-loop control signal: A similar analysis was done for the control signal of the admittance controller. We considered the control signal as the DQ acceleration $\underline{\boldsymbol{x}}_{r}$, since it represents the trajectory passed to the inner loop, and is directly related to the $\dot{\zeta}_{r d}^{r}$ through the relations (2), (1), $\underline{\boldsymbol{x}}_{d}^{r}=\underline{\boldsymbol{x}}_{r}^{*} \underline{\boldsymbol{x}}_{d}$, and their derivatives. Figs. 6a6c show the control effort $\sqrt{\int_{0}^{T}\left\|\ddot{\boldsymbol{x}}_{r}(t)\right\|^{2} d t}$, the maximum value of the control signal $\max _{t}\left(\left\|\ddot{\boldsymbol{x}}_{r}(t)\right\|\right)$, and its mean value

\footnotetext{
${ }^{8}$ The norm of the error has an exponential decay, but the same is not always true for each individually coefficient of the error. Therefore, the discrepancy function is defined as the difference between the error norms instead of the norm of the difference between the errors.
} 


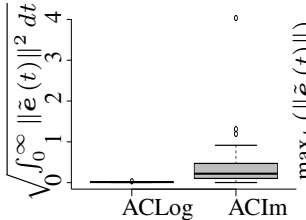

(a)

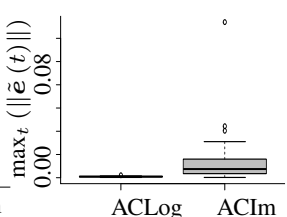

(b)

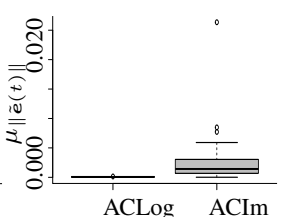

(c)
Figure 5: Error dynamics: (a) integral of the discrepancy function along the motion, (b) maximum value of the discrepancy function, and $(c)$ its mean for each controller.

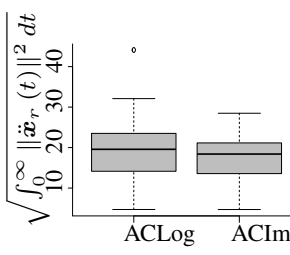

(a)

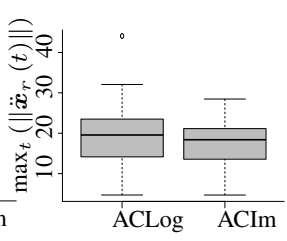

(b)

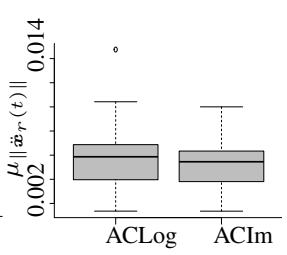

(c)
Figure 6: Outer-loop control signal: (a) control effort, (b) maximum value, and mean.

$\mu_{\left\|\ddot{\boldsymbol{x}}_{r}(t)\right\|}$, respectively. The hypothesis tests resulted in p-values equal to $0.328,0.328$, and 0.3817 for the effort, maximum, and mean values, which are all greater than the significance level $\alpha=0.05$. Therefore, the null-hypothesis cannot be rejected and there is no significant statistical difference between the control signals of the two controllers.

5) Statistical analyses of the inner loop control signal: The same analyses were made for the control signal of the kinematic controller, that is, control effort $\sqrt{\int_{0}^{T}\|\boldsymbol{u}(t)\|^{2} d t}$, maximum value of the control signal $\max _{t}(\|\boldsymbol{u}(t)\|)$, and its mean value $\mu_{\|\boldsymbol{u}(t)\|}$. The p-values for the Wilcoxon Rank Sum Test were $0.7412,0.7191$, and 0.8776 , for the control effort, maximum value, and mean value, respectively. Again, all the values were larger than $\alpha$, indicating that there is no significant statistical difference between the control signal of both controllers. This is expected because the reference signal for the inner loop is generated by the outer loop, and the outer loop generates statistically equivalent control signals for both ACLog and ACIm, as shown in Section IV-D4.

6) Analyses of special cases: Besides the statistical analyses with 30 different samples, we also analyzed four special cases:

1) the closed-loop system under the admittance controller ACIm starts in the unstable equilibrium set (i.e., when $\phi(0)=\pi)$;

2) the closed-loop system starts near this unstable equilibrium point;

3) a situation where the unwinding phenomenon appears $\left(\underline{\boldsymbol{x}}_{d}^{r}=-1\right)$

4) a situation where $\underline{\boldsymbol{x}}_{d}^{r}$ is closer to -1 than to 1 .

For case 1 , we considered an initial displacement of $\underline{\boldsymbol{x}}_{d}^{r}=\cos (\pi / 2)+\hat{k} \sin (\pi / 2)$, which consists of a pure rotation of $\pi$ around the $z$ axis. Fig. 7a shows that, whereas the error norm decays when using the ACLog, it remains unchanged for the ACIm, which is undesirable as the current pose is different from the desired one. This is due to the topological obstruction [5], which means that, when the initial state of

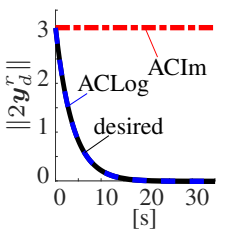

(a) Case 1

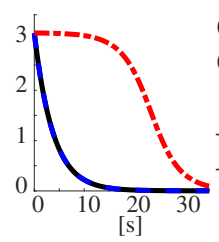

(b) Case 2

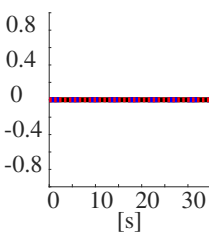

(c) Case 3

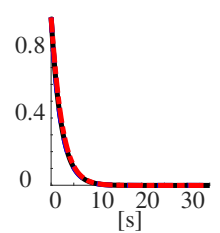

(d) Case 4
Figure 7: Closed-loop system during free-motion: time-evolution of the error norms in the four special cases.

the closed-loop system is within the unstable equilibrium set, the control signal is zero, trapping the system in that set. ${ }^{9}$ This situation is common in bimanual tasks, in which the endeffectors may be symmetric to the manipulated object, with an initial rotation angle of $\pi \mathrm{rad}$, and have to align themselves, such as the tasks of folding a sheet of paper, closing or opening a bottle, and also in tasks such as rotating a crank by $\pi \mathrm{rad}$. Although a small perturbation around the unstable equilibrium set may remove the system from it [1], case 2 occurs.

For case 2, we initialize the displacement near the unstable equilibrium point (i.e., $\left.\underline{\boldsymbol{x}}_{d}^{r}=\cos (\pi / 2-0.002)+\hat{k} \sin (\pi / 2-0.002)\right)$. In that case, the closed-loop error decays when using the ACIm, but much slower than when the ACLog is used, as shown in Fig. 7b, because the control signal near the unstable equilibrium point tend to be very small.

In case 3, the initial pose already equals the desired one, but the closed-loop system under the control law ACLog is in the PIS -1 . Fig. 7c shows that the error is always zero, indicating that the end-effector does not move for any controller. More specifically, the ACLog does not drive the system towards the PIS 1, which would make the end-effector move. Therefore, there is no unwinding.

Lastly, we considered $\underline{\boldsymbol{x}}_{d}^{r}=\cos (\pi+0.5)+\hat{k} \sin (\pi+0.5)$ to represent case 4 . In this case the error norm for both controllers decay at the same rate, which indicates that the end-effector is converging to -1 instead of 1 , performing the smallest path, as desired.

Remark 8. Simulation results show that when there is an external wrench applied at the end-effector under special cases $1-4$, both controllers behave analogously to when they are in free-motion. More specifically, ACIm suffers from the problem of topological obstruction and loses performance near the unstable equilibrium set, whereas ACLog neither suffers from topological obstruction nor unwinding. Those simulation results were omitted due to space constraints.

\section{E. Qualitative comparison between ACLog and ACIm}

Besides the problem of topological obstruction in the ACIm (25), which is not present in the ACLog (15), another difference in both formulations is the stiffness term. More

\footnotetext{
${ }^{9}$ To verify that behavior, consider (25) with $\boldsymbol{\varsigma}^{r}=\boldsymbol{\zeta}_{r d}^{r}(0)=\mathbf{0}, \phi=\pi$, $\boldsymbol{p}_{r d}^{r}=0$, and $\boldsymbol{n}_{r d}^{r} \in \mathbb{H}_{p} \cap \mathbb{S}^{3}$ such that $\boldsymbol{K}_{\phi} \operatorname{vec}_{3} \boldsymbol{n}_{r d}^{r}=\lambda_{\phi} \operatorname{vec}_{3} \boldsymbol{n}_{r d}^{r}$ with $\lambda_{\phi} \in(0, \infty)$. Then $\dot{\boldsymbol{\zeta}}_{r d}^{r}=-\boldsymbol{M}_{d}^{-1} \boldsymbol{K}_{d}^{\prime \prime} \boldsymbol{h}_{d}^{r}$, with $\boldsymbol{K}_{d}^{\prime \prime} \boldsymbol{h}_{d}^{r} \stackrel{r d}{=}$ $\left[\left(2 \lambda_{\phi} \boldsymbol{S}\left(\operatorname{vec}_{3} \boldsymbol{n}_{r d}^{r}\right) \operatorname{vec}_{3} \boldsymbol{n}_{r d}^{r}\right)^{T} \mathbf{0}_{1 \times 3}\right]^{T}=\mathbf{0}_{6 \times 1}$. Therefore, $\dot{\boldsymbol{\zeta}}_{r d}^{r_{d}}=\mathbf{0}$.
} 
specifically, because the stiffness term in the ACIm is given by

$$
\boldsymbol{K}_{d}^{\prime \prime} \boldsymbol{h}_{d}^{r}=\boldsymbol{K}_{d}^{\prime \prime}\left[\left(\operatorname{vec}_{3}\left(\boldsymbol{n}_{r d}^{r} \sin \left(\frac{\phi}{2}\right)\right)\right)^{T}\left(\operatorname{vec}_{3} \boldsymbol{p}_{r d}^{r}\right)^{T}\right]^{T},
$$

the term related to the orientation is nonlinear due to the sine function, differently from the stiffness term in the ACLog, which is linear in the orientation angle (see Remark 2). This non-linearity in the ACIm stiffness generates a nonexponential error decay, which may explain why the error discrepancy is larger for the ACIm, as shown in Fig. 5. Moreover, in case 2 of Section IV-D6, the error decay is slower for the ACIm than the ACLog due to the small values of the control signal close to the unstable equilibrium point.

\section{CONCLUSION}

This paper proposed a six-DOF coupled task-space admittance controller using dual quaternion algebra. Based on the energy of the system and on an appropriate algebraic representation, the proposed controller has a clear physical meaning and the stiffness term is consistent with the geometry of the 6-DOF task for arbitrary rigid motions. The use of unit DQ prevents the occurrence of representational singularities and, thanks to the use of the DQ logarithmic mapping, there are no trigonometric functions in the vector of motion displacement in the stiffness term, which improves the closed-loop error dynamics. Theoretical analyses, simulations, and experimental results show that, whereas one of the main controllers of the state of the art (ACIm) [2] suffers from topological obstruction, our switched controller (ACLog) does not. A thorough statistical analysis showed that when the ACLog is used, the closed-loop task error dynamics is closer to the desired specification than when the ACIm is used, whereas their control efforts are statistically equivalent.

\section{APPENDIX}

\section{A. Admittance control using the imaginary part of the rotation quaternion (ACIm)}

Caccavale et al. [2] proposed a similar controller as (14), but they used the imaginary part of a unit quaternion to represent the rotational displacement in the stiffness term. More specifically, their admittance controller is given by ${ }^{10}$

$$
\dot{\boldsymbol{\zeta}}_{r d}^{r}=\boldsymbol{M}_{d}^{-1}\left(-\boldsymbol{I}^{\#} \boldsymbol{\varsigma}^{r}-\boldsymbol{B}_{d} \boldsymbol{\zeta}_{r d}^{r}-\boldsymbol{K}_{d}^{\prime \prime} \boldsymbol{h}_{d}^{r}\right)
$$

where $\boldsymbol{h}_{d}^{r} \triangleq\left[\left(\operatorname{vec}_{3} \operatorname{Im}\left(\boldsymbol{r}_{d}^{r}\right)\right)^{T} \quad\left(\operatorname{vec}_{3} \boldsymbol{p}_{r d}^{r}\right)^{T}\right]^{T}$, with $\operatorname{Im}\left(\boldsymbol{r}_{d}^{r}\right)=\boldsymbol{n}_{r d}^{r} \sin (\phi / 2)$, and

$$
\boldsymbol{K}_{d}^{\prime \prime}=\left[\begin{array}{cc}
2 E^{\prime T}\left(\boldsymbol{r}_{d}^{r}\right) \boldsymbol{K}_{\phi} & \frac{1}{2} \boldsymbol{K}_{p}^{\prime \prime} \\
\mathbf{0}_{3 \times 3} & \boldsymbol{K}_{p}^{\prime}
\end{array}\right]
$$

with $E^{\prime}\left(\boldsymbol{r}_{d}^{r}\right)=\operatorname{Re}\left(\boldsymbol{r}_{d}^{r}\right) \boldsymbol{I}_{3 \times 3}-\boldsymbol{S}\left(\operatorname{vec}_{3} \operatorname{Im}\left(\boldsymbol{r}_{d}^{r}\right)\right)$ and $\operatorname{Re}\left(\boldsymbol{r}_{d}^{r}\right)=\cos (\phi / 2)$ [2]. As shown by Caccavale et al. [1], the closed-loop system has two sets of equilibrium points, one stable and the other one unstable. The latter consists

\footnotetext{
${ }^{10} \mathrm{We}$ changed the order of the rotational and translation terms in $\boldsymbol{h}_{d}^{r}$ and $\boldsymbol{\zeta}_{r d}^{r}$ to be consistent with our notation.
}

of rotations of $\pi \mathrm{rad}$ around a rotation axis parallel to any eigenvector of $\boldsymbol{K}_{\phi}$. If the initial state is inside this unstable set, the system gets trapped. This is the so-called topological obstruction [5].

\section{REFERENCES}

[1] F. Caccavale, C. Natale, B. Siciliano, and L. Villani, "Six-DOF impedance control based on angle/axis representations," IEEE Trans. Robot. Autom., vol. 15, no. 2, pp. 289-300, 1999.

[2] F. Caccavale, P. Chiacchio, A. Marino, and L. Villani, "SixDOF Impedance Control of Dual-Arm Cooperative Manipulators," IEEE/ASME Trans. Mechatronics, vol. 13, no. 5, pp. 576-586, oct 2008.

[3] B. Siciliano, L. Sciavicco, L. Villani, and G. Oriolo, Robotics: Modelling, Planning and Control, 1st ed., ser. Advanced Textbooks in Control and Signal Processing. London: Springer London, 2009.

[4] M. d. P. A. Fonseca, B. V. Adorno, and P. Fraisse, "Task-Space Impedance Controller Using Dual Quaternion Logarithm," in Work. Appl. Dual Quaternion Algebr. to Robot., 2019, pp. 1-2.

[5] S. Bhat and D. Bernstein, "A topological obstruction to global asymptotic stabilization of rotational motion and the unwinding phenomenon," in Proc. 1998 Am. Control Conf. ACC (IEEE Cat. No.98CH36207), vol. 5, no. June. IEEE, 1998, pp. 2785-2789 vol.5.

[6] H. T. Kussaba, L. F. Figueredo, J. Y. Ishihara, and B. V. Adorno, "Hybrid kinematic control for rigid body pose stabilization using dual quaternions," Journal of the Franklin Institute, vol. 354, no. 7, pp. 2769 2787, may 2017.

[7] B. V. Adorno, Robot Kinematic Modeling and Control Based on Dual Quaternion Algebra - Part I : Fundamentals, 2017. [Online]. Available: https://hal.archives-ouvertes.fr/hal-01478225/document

[8] _ "Two-arm Manipulation: From Manipulators to Enhanced HumanRobot Collaboration [Contribution à la manipulation à deux bras : des manipulateurs à la collaboration homme-robot]," Ph.D. dissertation, Université Montpellier II, 2011.

[9] L. F. C. Figueredo, B. V. Adorno, J. Y. Ishihara, and G. A. Borges, "Robust kinematic control of manipulator robots using dual quaternion representation," in Proc. - IEEE Int. Conf. Robot. Autom. Karlsruhe: IEEE, 2013, pp. 1949-1955.

[10] H. J. Savino, L. C. Pimenta, J. A. Shah, and B. V. Adorno, "Pose consensus based on dual quaternion algebra with application to decentralized formation control of mobile manipulators," J. Franklin Inst., vol. 357, no. 1, pp. 142-178, jan 2020.

[11] D. S. Bernstein, Matrix mathematics: theory, facts, and formulas, 2nd ed. Princeton University Press, 2009.

[12] C.-T. Chen, Linear System Theory and Design, 3rd ed., A. S. Sedra and M. R. Ligthner, Eds. New York: Oxford University Press, 1999.

[13] M. W. Spong, S. Hutchinson, and M. Vidyasagar, Robot Modeling and Control. John Wiley \& Sons, Inc, 2006.

[14] N. Hogan, "Impedance Control: An Approach to Manipulation," J. Dyn. Syst. Meas. Control, vol. 107, no. 1, pp. 1-24, 1985.

[15] J. J. E. Slotine and W. Li, Applied Nonlinear Control. Prentice Hall, 1991.

[16] R. Kelly, V. Santibáñez, and A. Loría, Control of Robot Manipulators in Joint Space. Leipzig: Springer, 2005.

[17] J. J. Quiroz-Omana and B. V. Adorno, "Whole-Body Control With (Self) Collision Avoidance Using Vector Field Inequalities," IEEE Robot. Autom. Lett., vol. 4, no. 4, pp. 4048-4053, 2019.

[18] B. V. Adorno and M. M. Marinho, "DQ Robotics: a Library for Robot Modeling and Control Using Dual Quaternion Algebra," no. 19, pp. 1-10, 2019. [Online]. Available: http://arxiv.org/abs/1910.11612

[19] B. Navarro, "Solutions for safe human-robot collaboration," PhD, Université d'Orléans, 2017.

[20] M. Hollander, D. A. Wolfe, and E. Chicken, Nonparametric Statistical Methods, 2nd ed., ser. Wiley Series in Probability and Statistics. Wiley, jul 1999. 